Review Article

\title{
Pediatric Multiple Sclerosis and Cognition: A Review of Clinical, Neuropsychologic, and Neuroradiologic Features
}

\author{
Ozgul Ekmekci \\ Department of Neurology, Faculty of Medicine, Ege University, Izmir, Turkey \\ Correspondence should be addressed to Ozgul Ekmekci; ozgul.ekmekci@ege.edu.tr
}

Received 6 July 2017; Revised 10 November 2017; Accepted 4 December 2017; Published 25 December 2017

Academic Editor: Lambros Messinis

Copyright (c) 2017 Ozgul Ekmekci. This is an open access article distributed under the Creative Commons Attribution License, which permits unrestricted use, distribution, and reproduction in any medium, provided the original work is properly cited.

\begin{abstract}
Multiple sclerosis (MS) is an inflammatory demyelinating and neurodegenerative disease. Although cognitive impairment has been well established in adult patients with MS, its occurrence in patients with pediatric-onset MS has recently been reported. In this review, I discuss the main features of cognitive impairment in pediatric MS as determined by long-term follow-up studies, neuropsychiatric test batteries, and the results of neuroradiological imaging studies that investigated the pathogenesis of pediatric MS. The most commonly affected cognitive domains in adults are attention, processing speed, and visuomotor skills; language and intelligence are also affected in pediatric MS. A young age at disease onset is the strongest risk factor for these impairments, which may be due to the effect of inflammatory demyelination and neurodegeneration on the developing central nervous system and neural networks in children. Cognitive impairment has long-term effects on patients' academic life and the quality of their social life. Therefore, all patients with pediatric MS should be screened and monitored for cognitive impairment. This review also highlights the need for neuropsychological test batteries that assess different cognitive domains in children and adolescents with multiple sclerosis and for cognitive rehabilitation programs to improve the quality of their academic and social life.
\end{abstract}

\section{Introduction}

Multiple sclerosis (MS) is an autoimmune-mediated neurodegenerative and demyelinating disorder of the central nervous system primarily affecting young adults. Approximately $2-5 \%$ of MS patients have onset before age $18[1,2]$. The International Pediatric Multiple Sclerosis Study Group defined diagnostic criteria for pediatric MS [3]. MS can cause both physical and cognitive disability. The occurrence of cognitive impairment is well established in adult patients with MS, occurring in approximately $45-65 \%$ of these patients [4]. The cognitive domains that are most commonly affected in adult MS patients are complex attention, information processing speed, episodic memory, visuospatial abilities, and executive functions [5]. Cognitive impairment in pediatric MS patients has been described with different studies reporting cognitive impairment in 30-50\% of cases [5-8]. Cognitive impairment in pediatric multiple sclerosis differs from that in adults in terms of the cognitive domains involved. Factors such as age, maturation of the central nervous system, and cognitive reserve have different effects on cognition in childhood. The maturation status of the central nervous system at the time of MS onset is a long-term prognostic factor, and it is crucial to develop appropriate neuropsychological test batteries that assess different cognitive domains in children and adolescents with MS. Moreover, cognitive rehabilitation to improve the quality of academic and social life in pediatric MS patients has not yet been well studied. In this review, I discuss the main features of cognitive impairment in pediatric MS, long-term follow-up findings, neuropsychiatric test batteries, and the result of neuroradiological imaging studies that investigated the pathogenesis of pediatric MS.

\section{The Main Features of Cognitive Impairment in Pediatric MS Patients}

Banwell and Anderson were the first to use a neuropsychological battery to systematically investigate a cohort of patients with pediatric MS. They assessed cognitive functions in 10 children with MS and identified deficits in general cognition, language, visuomotor integration, and verbal and visual memory. Children with longer disease duration demonstrated 
deficits in executive functions, information processing speed, and working memory, suggesting that children with longer disease duration and a younger age at onset are at higher risk for cognitive impairment [6].

MacAllister et al. evaluated 37 children and adolescents with MS and reported significant cognitive impairment in $35 \%$ of these patients. The most common impairment was in complex attention. They also found receptive language problems and poor naming in many patients, whereas verbal fluency was unaffected. Immediate verbal memory was impaired in only one patient; however, delayed recall was impaired in seven patients. The researchers suggested that, in these patients, the effect of MS on attention and memory was similar to that in adults with MS, but visuospatial functions were less affected. They found a strong relationship between cognitive impairment and Expanded Disability Status Scale (EDSS) score, total number of relapses, and disease duration [7].

In a multicenter study, Amato and colleagues assessed the frequency, pattern, and clinical correlates of cognitive impairment in childhood and adolescent MS cases and compared these characteristics with those in healthy controls. The researchers used a neuropsychological battery to assess intelligence quotient (IQ), memory, attention/concentration, executive functions, and language. They found significant cognitive impairment in $31 \%$ of the patients with MS, and $53 \%$ of the cases failed at least two tests. Both verbal and performance IQ scores were lower in MS patients than in healthy controls. The most frequently affected cognitive domains were memory, particularly visuospatial memory, complex attention, verbal comprehension, and executive functions. In this study, EDSS and number of relapses were not adequate descriptors of cognitive condition; cognitive impairments were found also in patients with low disability levels. In this cohort, there was a significant correlation between cognitive impairment and low IQ scores. Low IQ score was also significantly associated with younger age at disease onset [8].

In a study of the magnetic resonance imaging (MRI) correlates of cognitive impairment in pediatric MS patients, Till et al. found impairments in attention and processing speed, visuomotor integration, and expressive language. They observed cognitive impairment in $29.4 \%$ of the MS patients. Full-scale IQ and verbal IQ were significantly reduced in children with MS and a higher IQ was associated with a shorter disease duration and an older age at disease onset. Moreover, a higher full-scale IQ was correlated with a lower EDSS score, and patients with cognitive impairment tended to be male. Finally, cognitive impairment was associated with a longer disease duration and a younger age at onset [9].

In a large multicenter study, Julian et al. examined cognitive function in 231 pediatric patients with MS $(n=187)$ or clinically isolated syndrome $(n=44)$. The cognitive impairment was noted in $35 \%$ of patients with MS and $18 \%$ of patients with clinically isolated syndrome. The cognitive areas that were most frequently affected were fine motor coordination (54\%), visuomotor integration (50\%), and information processing speed (35\%). EDSS score was the only variable that was significantly and independently associated with reduced cognitive function [10].

The methods and results of these cross-sectional studies are shown in Table 1. Although these studies used different neuropsychological tests and different definitions of cognitive impairment, they found similar rates of cognitive impairment. The rate of cognitive impairment in pediatric MS ranged from $29.4 \%$ to $35 \%$. Full-scale IQ and verbal and performance IQ were lower in children with MS. Low IQ scores were associated with higher EDSS score and young age at disease onset $[8,9]$. The relationship of cognitive impairment with young age at onset and disease duration was similar in many studies [6-9]. However, the relationship between EDSS score and cognitive impairment varied between these studies. For example, Julian et al. and Banwell and Anderson found that EDSS score was associated with cognitive impairment but other studies did not $[6,10]$. These studies suggested that cognitive impairment affected academic and social activities negatively and impaired the quality of life [6-8].

In summary, similar to adult patients, pediatric MS patients experience deficits in attention, information processing speed, memory, executive functions, and visuomotor integration. Additionally, unlike in adults, MS affects language functions and intelligence occurs in particularly young children. The relationship between cognitive dysfunction and disease duration, disability scores, and relapse frequency is unclear. The strongest predictor is a younger age at disease onset. Although the relationship between motor disability and cognitive disability is controversial, cognitive impairment could occur independently from motor disability. Cognitive impairment affects academic and social activities and quality of life. Monitoring of cognitive impairment in pediatric MS patients is important to enable optimal treatment and rehabilitation for prevention of further cognitive disability.

Another factor affecting MS-induced cognitive functions is cognitive reserve. The cognitive reserve theory states that individual differences in how tasks are processed or neural networks are used can allow some people to cope better with brain pathology than others [11]. It has been shown that both heritable (brain reserve) and environmental (greater intellectual enrichment) factors may attenuate the negative effects of MS on cognitive status in adults [12]. The role of cognitive reserve in pediatric MS is unclear. Pastò et al. assessed the potential impact of cognitive reserve on cognition in a cohort of pediatric MS patients. A total of 48 pediatric MS patients and 57 healthy controls were included in this study, and patients were followed up for $4.7 \pm 0.4$ years. Cognitive reserve was estimated by measuring the IQ at baseline, and cognitive impairment was defined as failure in $\geqslant 3$ tests of an extensive neuropsychological battery that included the Selective Reminding Test (SRT) and Selective Reminding Test-Delayed (SRT-D), the 10/36 Spatial Recall Test (SPART), the 10/36 Spatial Recall Test-Delayed, the Symbol Digit Modalities Test (SDMT), the Trail Making tests (TMT-A and TMT-B), a Semantic and Phonemic Verbal Fluency Test, an Oral Denomination Test from the Aachener Aphasia Test, the Token Test, the Indication of Pictures from the Neuropsychological Examination for Aphasia, and Phrase Comprehension Test from the Battery for the 


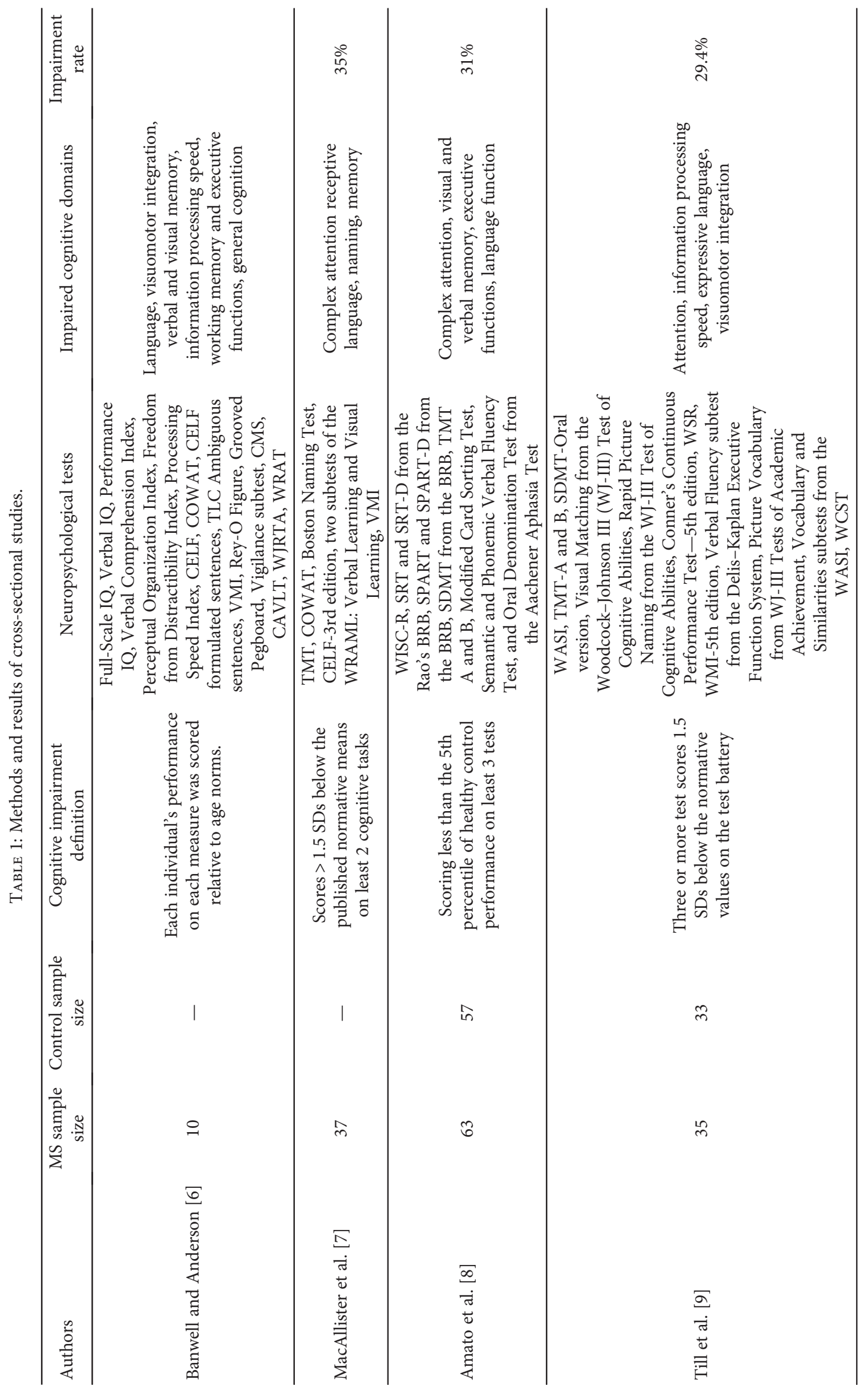




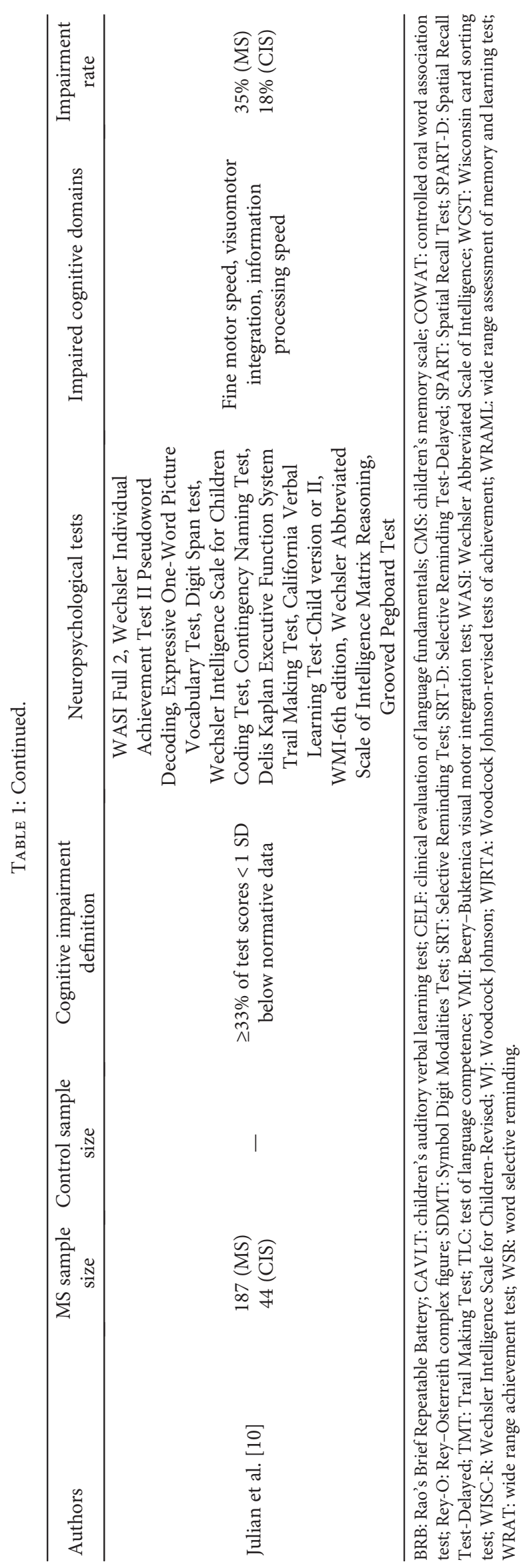


Analysis of Aphasic Deficits. The reliable change index (RCI) method was used to assess changes in neuropsychological performance. Cognitive impairment was found in $29.2 \%$ of patients with pediatric onset MS at baseline. Deterioration in cognitive performance was detected using the RCI method in $37.6 \%$ patients with pediatric onset MS. A higher cognitive reserve predicted stable or improving performance, whereas a lower reserve was associated with deteriorating performance at follow-up [13]. In other studies, on traumatic brain injury, higher cognitive reserve was associated with higher performance and a greater resistance to the development cognitive deficits [13-15]. Thus, measurement of cognitive reserve can be used to identify patients at high risk for cognitive impairment. Alternatively, cognitive reserve can be used to develop cognitive-protective approaches and cognitive rehabilitation programs.

Compared to adults, children are better able to compensate for brain damage because of the greater neural plasticity of the developing brain. Pediatric MS occurs during periods of brain growth, myelination, and neural network maturation; therefore, longitudinal follow-up studies are important to understanding the interactions between progressive brain pathology and maturation, neural plasticity, and compensatory abilities. During 2- and 5-year follow-ups of childhood and adolescent MS cases in their multicenter study, Amato et al. used the same neuropsychological battery as that used at baseline, with alternative versions of the tests to assess memory, attention/concentration, executive functions, and language. At the 2-year follow-up, $70 \%$ of patients failed at least three tests, and overall, $75 \%$ of the cases were classified as having deteriorating cognitive performance. Failure was more prominent in tests of verbal memory, complex attention, verbal fluency, and receptive language. An individual cognitive change index was also calculated to assess the direction of change in cognition over the follow-up period. At the 2-year follow-up, cognitive deterioration was observed in $75 \%$ of the patients, while $25 \%$ of the cases were classified as stable or improving. At the 5-year follow-up, the cognitive impairment index decreased in 56\% of the patients, improved in $25 \%$, and remained stable in $18.8 \%$. Cognitive deterioration was associated with male sex, younger age at disease onset, and lower educational level. The cognitive domains with the most tendencies toward deterioration were visuospatial learning and expressive language $[16,17]$.

In a small cohort of patients with pediatric MS, MacAllister et al. assessed cognitive function longitudinally and found deficits in attention, executive functions, and memory. Their findings suggested that neurological impairment early in the course of the disease was predictive of cognitive decline over time. There was no strong association between duration of disease and cognitive decline. The results suggested that, in patients with pediatric MS, cognition might deteriorate over time relative to that of their peers and their baseline performance [18].

In a longitudinal study, Charvet et al. evaluated cognitive function in 62 patients with pediatric MS and five with clinically isolated syndrome. The researchers evaluated cognition at baseline and at follow-up, with a mean follow-up time of
$1.64 \pm 0.63$ years. Cognitive impairment was detected in $37 \%$ of patients at baseline and $33 \%$ of patients at followup. The most commonly impaired cognitive functions included visuomotor integration, information processing speed, and attention. The researchers indicated that cognitive performance might remain stable, or even improve, over a short period of time [19].

In another longitudinal study, Hosseini et al. investigated how the age at disease onset and cognitive reserve may affect cognitive maturation in children and adolescents with pediatric MS. They used two standardized and widely used neuropsychological measures (SDMT and TMT) to assess visual scanning, information processing speed, and working memory. Cognitive reserve capacity was assessed using the patient's baseline full-scale IQ and parental social status. They used growth curve analysis to evaluate cognitive maturation over childhood and adolescence. Their results showed that a younger age at disease onset was associated with decreased performance on neuropsychological measures (SDMT and TMT). A younger age of disease onset was also associated with lower developmental gain on the neuropsychological measures. Cognitive reserve, including baseline full-scale IQ and parental social status, did not protect against cognitive deterioration [20].

Till et al. investigated the extent, pattern, and correlates of change in cognitive functioning over an approximately 15-month interval in 28 patients with pediatric MS and compared their findings with data from 26 healthy controls. The RCI was used to determine individual differences on test scores over time in this prospective longitudinal study. Participants were categorized as showing either "decline" or "improvement" if changes in scores exceeded the RCI on three or more tests. They observed that healthy controls were more likely to show improvement across multiple domains of function than patients with pediatric MS were. When cognitive change was examined on an individual basis, cognitive deterioration was observed in seven of 28 patients with pediatric MS and only one of 26 healthy controls. Clinically significant improvement on three or more of the neuropsychological tests was detected in $69.2 \%$ of healthy controls compared with only $17.9 \%$ of pediatric MS patients. Patients with pediatric MS showed decline mainly on tests of information processing speed and attention, visuomotor integration, verbal fluency, abstract visual memory, calculation, and spelling. An association was found between disease duration and deterioration in visuomotor integration. The results showed that patients who were classified as having declined cognitive function $(n: 7)$ did not differ from those with stable/improved cognitive function $(n: 21)$ with regard to age at testing, age at disease onset, disease duration, sex, mood-related symptoms, baseline IQ, and EDSS scores. The researchers reported that patients with pediatric MS failed to demonstrate age-expected improvements in several domains of cognitive performance [21].

In a study investigating long-term outcomes in adult patients with pediatric-onset MS, SDMT scores were lower in patients with pediatric onset than adult onset both in unadjusted analysis and after adjustment for disease 
duration. The researchers suggested that, in adulthood, pediatric-onset MS may result in greater impairment in processing speed and that further investigation of other cognitive domains was warranted in this group of patients [22].

The methods and results of longitudinal follow-up studies are shown in Table 2. The duration of follow-up in these studies varied between 1 and 5 years. In Amato et al.'s study, most of the patients (75\%) showed deteriorating performance between baseline and year 2; however, comparing baseline and 5-year testing, cognitive impairment index deterioration was observed in $56 \%$ of the patients, improvement in $25 \%$, and stability in $18.8 \%[16,17]$. Charvet et al. emphasized that most of the cases had a stable cognitive function in the mean 1.6-year follow-up [19]. Till et al. found that $75 \%$ of the cases showed stabilization or improvement in cognitive function at 15 months follow-up, but the improvement rate was less than that of healthy controls [21]. The variations in the results of the studies may be related to the differences in follow-up periods and evaluation methods used.

The reason for the improvements observed in children may be the ongoing development in the brain and neural plasticity. Brain development and neural plasticity may be involved in the success of cognitive rehabilitation and cognitive reserve enhancement approaches. Alternatively, cognitive performance in patients with pediatric MS may deteriorate in the long term relative to their baseline performance and the performance of their peers. This worsening cognitive state can be explained by impairment of the maturation and development of the immature central nervous system by demyelinating and neurodegenerative processes.

\section{Social Cognition}

Social cognition, a distinct area of cognitive function, refers to "the mental operations that underlie social interactions, including perceiving, interpreting, and generating responses to the intentions, dispositions, and behaviors of others" [23]. The theory of mind is an essential domain of social cognition, defined as the ability to infer other people's mental states, including beliefs, desires, knowledge, and intentions, hence explaining and predicting their behavior [24]. Social cognitive impairment has been studied in autism, psychiatric disorders, and developmental and neurodegenerative diseases. However, studies on social cognition in pediatric MS are very limited, with only one study published in the literature. Charvet and colleagues examined social cognition in 28 patients with pediatric-onset MS and 38 healthy controls. They used the SDMT and all three theories of mind tasks: reading the mind in the eyes test, the faux pas test, and the false-belief task. Patients with pediatric-onset MS performed worse than healthy controls on all theories of mind tasks, including both affective (eyes test) and cognitive (faux pas test and false-belief task) measures. Information processing speed, which was measured by SDMT, was slower in the pediatric-onset MS group (38\%) than in the healthy control group (6\%). However, when logistic regression analysis was performed to assess the influence of information processing speed on the theory of mind tasks, they suggested that information processing speed had no influence on the theory of mind [25]. Social cognitive impairment has been reported in adult patients with MS who were otherwise cognitively intact $[26,27]$. Social cognition is important for the ability to form and maintain personal relationships. The effect of MS on social cognition is particularly important in patients with pediatric-onset MS since their social cognition skills are still undergoing development [25-27]. More studies on social cognition in pediatric MS are needed for the identification and prevention of social adjustment problems in these patients.

\section{Neuropsychological Test Batteries for Pediatric MS}

The aforementioned studies applied neuropsychological tests developed for adults to pediatric patients with MS. There is a need for a standardized, well-approved neuropsychological battery to detect MS-related deficits in pediatric patients. It is important to establish neuropsychological test batteries for evaluating cognitive domains that appear more frequently in children than adults and for assessing cognitive maturation in this age group. Several studies have attempted to establish such a neuropsychological test battery and to measure the sensitivity of presently available tests.

In an Italian multicenter study, Portaccio et al. validated a brief neuropsychological battery that they derived from the extensive battery used by Amato and colleagues. The researchers aimed to develop a Brief Neuropsychological Battery for Children (BNBC) with MS. The extensive neuropsychological battery involved the Wechsler Intelligence Scale for Children-Revised (WISC-R), the Selective Reminding Test (SRT, SRT-Delayed) from Rao's Brief Repeatable Battery (BRB), the Spatial Recall Test (SPART, SPART-D) from the BRB, the Symbol Digit Modalities Test (SDMT) from the BRB, Trail Making Test (TMT-A and TMT-B), Modified Card Sorting Test, Semantic Verbal Fluency Test (SVFT), Phonemic Verbal Fluency Test (PVFT), the Oral Denomination Test from the Aachener Aphasia Test, Token Test, the Indication of Pictures from the Neuropsychological Examination for Aphasia, and the Phrase Comprehension Test from the Battery for the Analysis of Aphasic Deficits (Table 3). Cognitive functions were assessed in 61 patients with childhood and juvenile MS and in 58 healthy controls. In this study, cognitive impairment was found in $41 \%$ of MS patients. Verbal and spatial memory (SRT and SPART), attention and concentration (SDMT and TMT), and language abilities (SVFT and Token Test) were the most frequently affected cognitive domains. Discriminant function analysis showed that the SDMT, the TMT-B, the Selective Reminding Test-Consistent Long-Term Retrieval (SRT-CLTR), and the vocabulary test from the WISC had higher discriminating ability. Therefore, using these tests, the researchers created the BNBC, which had a sensitivity of $96 \%$ and a specificity of $76 \%$ [28]. (Table 4).

Smerbeck et al. investigated the sensitivity and validity of two visual processing tests in 43 children with pediatric MS and 45 healthy controls: the Brief Visuospatial Memory Test-Revised (BVMT-R) and the SDMT. Previous large cross-sectional studies had demonstrated the sensitivity of 


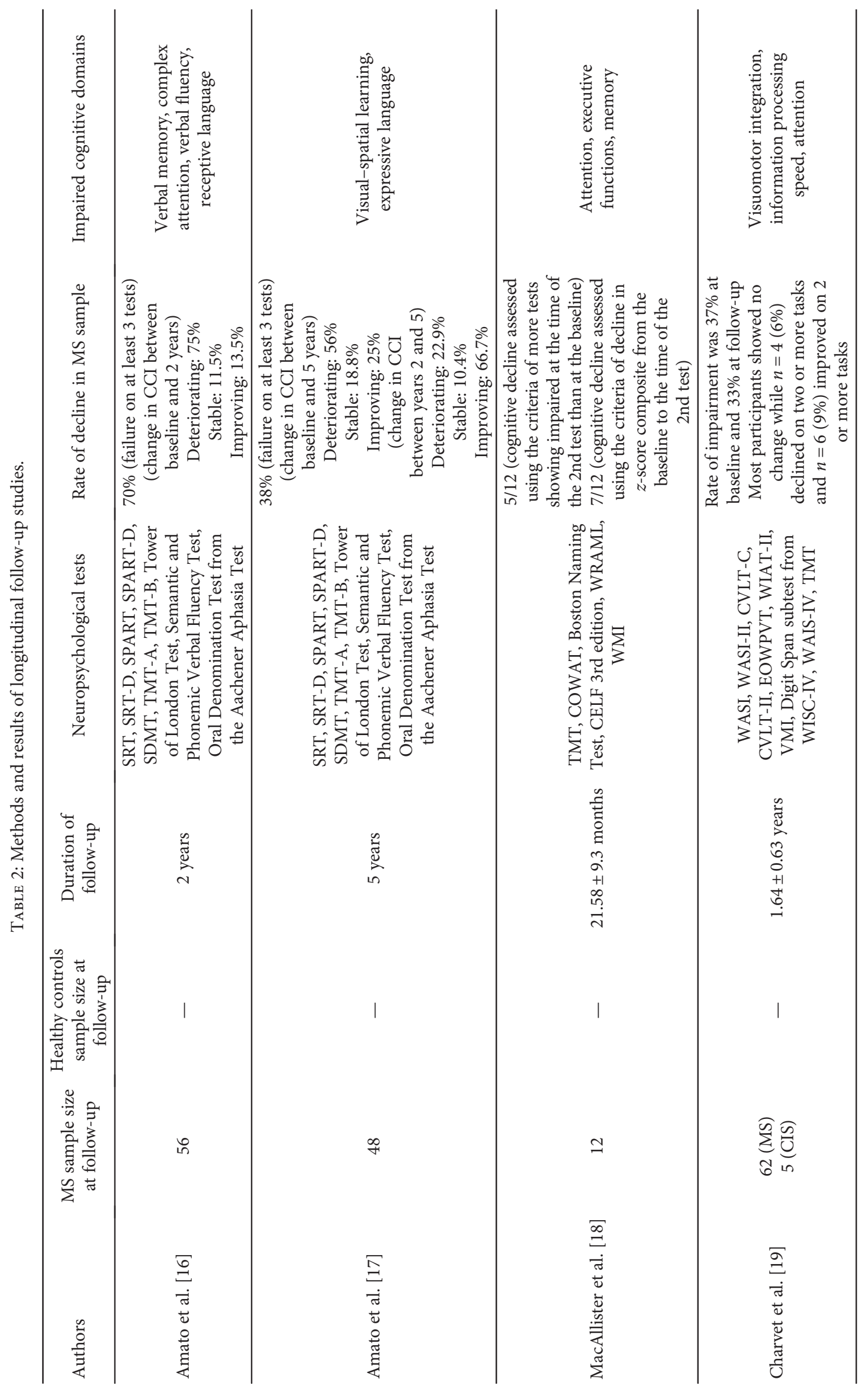




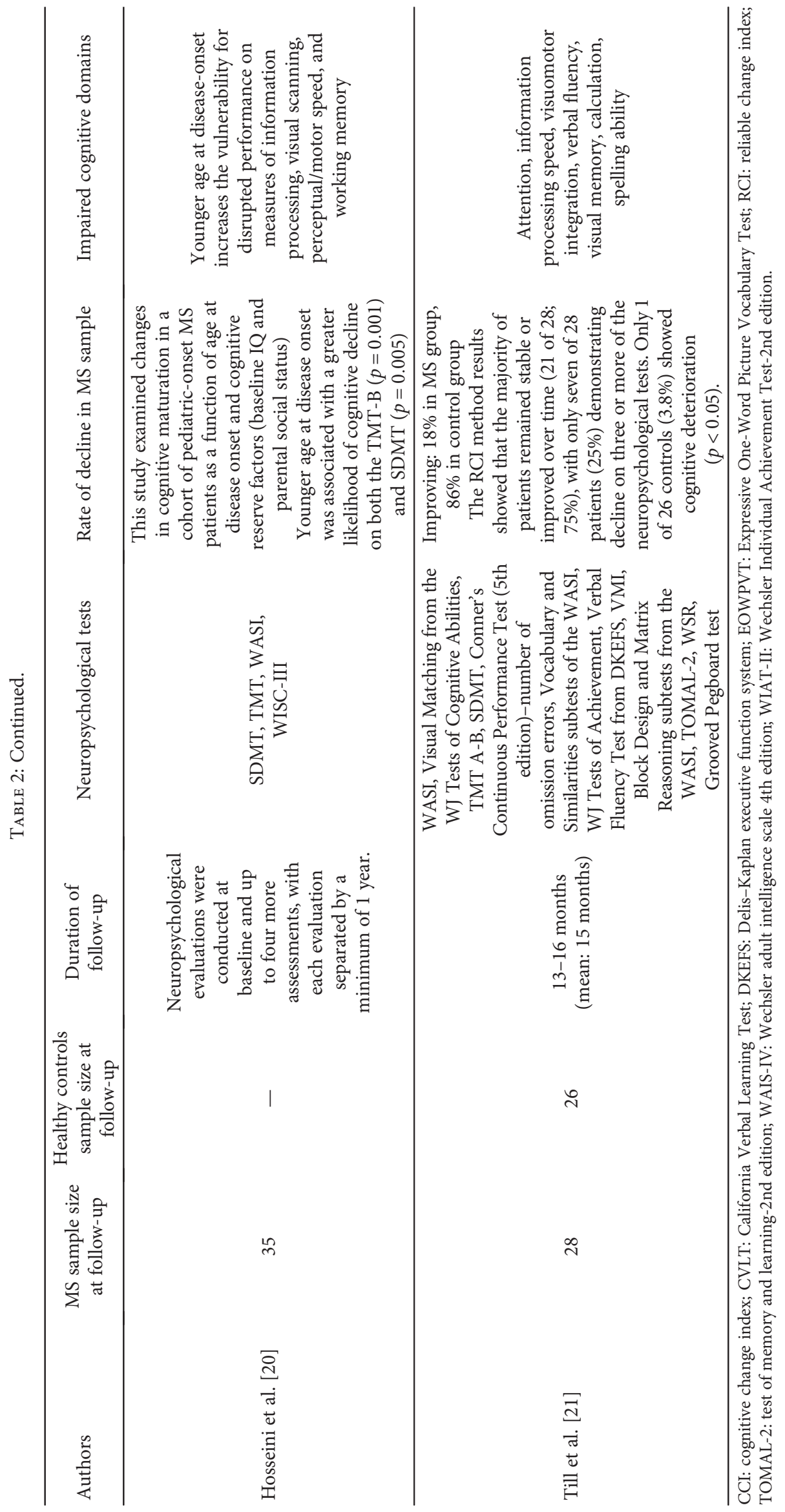


TABLE 3: Neuropsychological tests.

\begin{tabular}{lc}
\hline Neuropsychological test & Cognitive domain \\
\hline WISC-R (Wechsler Intelligence Scale for Children) & IQ (intelligence quotient) \\
\hline SRT-LTS (Selective Reminding Test-Long-Term Storage) & Memory \\
SRT-CLTR (Selective Reminding Test-Consistent Long-Term Retrieval) & SRT-D (Selective Reminding Test-Delayed) \\
SPART (Spatial Recall Test) & Abstract/conceptual reasoning \\
SPART-D (Spatial Recall Test-Delayed) & Attention/concentration \\
\hline MCST (Modified Card Sorting Test) & Language \\
\hline SDMT (Symbol Digit Modalities Test) & \\
TMT-A/ B (Trail Making Test A/B) & \\
\hline SVFT (Semantic Verbal Fluency Test) & \\
PVFT (Phonemic Verbal Fluency Test) & \\
IPT (Indication of Pictures Test) & \\
PCT (Phrase Comprehension Test) & \\
ODT (Oral Denomination Test) &
\end{tabular}

TABLE 4: Brief Neuropsychological Battery for Children (BNBC).

(i) SRT
(ii) SDMT
(iii) TMT
(iv) Vocabulary test from the WISC-R

Duration: 30 minutes. Sensitivity (96\%), specificity (76\%).

these tests in adults. They found statistically significant differences between children with MS and healthy controls on BVMT-R Total Learning, BVMT-R Delayed Recall, and SDMT. These findings indicated that two relatively brief measures of visual-cognitive processing could be successfully applied to the pediatric population and could be useful in detecting and monitoring significant cognitive impairment [29].

Charvet et al. evaluated the SDMT as a screening tool for identifying pediatric onset MS patients at risk for cognitive impairment. The SDMT showed $77 \%$ sensitivity and $81 \%$ specificity for detecting neuropsychological impairment when administered within 1 year. Age and EDSS score were negatively correlated with SDMT score. The researchers concluded that these results support the use of the SDMT as a screening tool for cognitive function in pediatric MS [30].

\section{Neuroradiologic Studies and Anatomic Correlation of Cognitive Impairment in Pediatric MS Patients}

Several neuroradiologic studies have reported a correlation between deficits in executive function and atrophy of the thalamus and frontal lobes, as well as the correlation of a decrease in cognitive speed and mathematical performance with corpus callosum damage [31-33].

To normalize total and regional brain volumes for head size in their MRI correlations, Till and colleagues calculated cortical gray matter, thalamic, and global brain volumes using a scaling factor computed using the normalization of atrophy method. In addition, they also calculated T1- and T2-weighted lesion volumes in MS patients. Thirty-five pediatric-onset MS patients and 33 healthy controls were included in the study. Thalamic volume, corpus callosum area, normalized brain volume, and normalized gray matter volume were significantly lower in patients with MS than in healthy controls. Thalamic volume was strongly and positively correlated with an index global intellectual function in MS patients. MS patients with cognitive impairment did not differ from MS patients without cognitive impairment with regard to T1 or T2 lesion volume, normalized grey matter volume, or normalized brain volume. Neuropsychologic test performance was highly correlated with global and regional brain volume and less strongly correlated with $\mathrm{T} 1$ and T2 lesion volumes. The researchers suggested that cognitive impairment was related to the neurodegenerative component of MS [9].

The cerebellum is another anatomical area associated with cognitive function. It is a strategic node in various networks such as coordination and cognitive-behavioral loops [34]. Weier et al. examined associations between cognitive outcomes and cerebellar volume independent of cerebral volume in patients with pediatric MS. Twenty-eight pediatric-onset MS patients and 33 age- and sex-matched healthy controls were included in this study. All subjects underwent structural MRI and neuropsychological evaluation to assess intelligence, attention, information processing speed, language, visuomotor integration, and fine-motor dexterity. Neuropsychological battery included the Wechsler Abbreviated Scale of Intelligence (WASI), the TMT-B, the SDMToral version, Beery Visual Motor Integration, the vocabulary subtest of the WASI, and the Grooved Pegboard test. Cognitive performance in patients with MS was reduced relative to that in the healthy controls in the domains of attention, information processing speed, expressive language, visuomotor integration, and fine motor dexterity. Cerebellar volumes did not differ between MS group and healthy controls; however, cerebellar posterior lobe volume and infratentorial 
lesion volume accounted for extra variance in cognitive measures of information processing (SDMT) and vocabulary within MS patients in regression analysis. The researchers suggested that in addition to cerebral volume, the cerebellar volume was another correlate of cognitive function in children and adolescents with MS [35].

In a neuroradiologic study, Rocca and colleagues performed a structural and functional MRI examination to investigate the mechanisms responsible for the presence and severity of cognitive impairment in pediatric patients with MS. A total of 35 pediatric patients with MS and 16 sex- and age-matched healthy controls were included in the study. The researchers used voxel-based analysis with advanced structural MRI techniques to determine the patterns of regional involvement of white and gray matter according to patient's cognitive profile. They also used functional MRI to quantify the resting-state functional connectivity of the default mode network (DMN). The BNBC was used to assess participants' cognitive function, and subjects with abnormal performance in two tests were classified as cognitively impaired. Among the patients with MS, $45 \%$ were classified as cognitively impaired. Spatial and verbal memory abilities, language, attention, and concentration were the most significantly involved cognitive areas. The results showed that cognitively impaired patients with MS had a higher occurrence of T2 lesions and white and gray matter damage, including atrophy and diffusivity abnormalities in the posterior region of the parietal lobes close to midline (precuneus, posterior cingulum, and corpus callosum). Reduced resting-state functional connectivity in the precuneus was observed in cognitively impaired patients, whereas cognitively preserved patients showed increased restingstate functional connectivity in the anterior cingulate cortex. The researchers concluded that the presence and severity of cognitive impairment were associated with structural and functional abnormalities of the posterior core regions of the DMN in pediatric patients with MS [36].

In these neuroradiological studies, researchers used different parameters such as global brain volume, thalamic volume, gray matter volume, T1-T2 lesion volume, and cerebellar volume. Cognitive impairment has been related to T2 hyperintense lesions, diffuse white matter damage, and cortical and deep gray matter atrophy in adult MS patients [37]. Neuropathologic findings have revealed that MS affects regions that are functionally or anatomically involved in cognitive processes, such as cortical and deep gray matter, hippocampus, white matter, including normal appearing white matter in adult patients [38]. Consistent with the findings in adult patients, studies in pediatric MS patients suggest that a relationship exists between cognitive impairment and gray matter atrophy, white matter atrophy, and global and regional brain volume $[9,36]$.

\section{Conclusions}

A third of the pediatric MS patients experience cognitive impairment. In pediatric cases, the influence of demyelination on the developing central nervous system and neural network affects cognitive function negatively; on the other hand, neural plasticity and ability of compensation can have a positive effect on cognitive functions. Unlike in adults, intelligence and language functions are affected in pediatric MS. Young age at disease onset increases the risk of cognitive impairment. Although varying findings have been reported for the relationship between motor disability and cognitive disability, cognitive impairment may also occur independently from motor disability. Cognitive reserve is important both for the detection of patients with high risk and for the development of cognitive-protective approaches and rehabilitation. Knowledge about social cognition is limited in pediatric MS. Cognitive impairment in pediatric MS is a critical problem that has long-term effects on patients' academic and social quality of life; thus, the development of appropriate neuropsychological test batteries is important for screening patients who are at risk. Current studies have shown that SDMT and Brief Neuropsychological Battery for Children (BNBC) could be effectively used for screening cognitive functions in pediatric MS.

MRI studies have suggested that cognitive impairment in pediatric MS can be related to both gray matter atrophy and white matter atrophy and global and regional brain volume. More longitudinal studies with standardized neuropsychological batteries and advanced MRI techniques are needed to explore the mechanism of cognitive impairments and predict high-risk group. Understanding the pathogenesis of impaired cognition and the involvement of specific cognitive areas in pediatric MS will guide the development of treatment and rehabilitation, including cognitive rehabilitation, about which limited data are available in pediatric MS patients.

Assessment of cognitive function is also important for the evaluation of response to treatment. While defining the response to treatment and disease activity in pediatric MS, preservation of age-expected global and regional brain growth and age-expected cognitive maturation and function should be assessed, as well as the number of relapses, disability progression, and MR findings such as new, enlarging, or enhancing lesions.

\section{Conflicts of Interest}

The author declares that there is no conflict of interest regarding the publication of this paper.

\section{References}

[1] P. Duquette, T. J. Murray, J. Pleines et al., "Multiple sclerosis in childhood: clinical profile in 125 patients," The Journal of Pediatrics, vol. 111, no. 3, pp. 359-363, 1987.

[2] A. Ghezzi, V. Deplano, J. Faroni et al., "Multiple sclerosis in childhood: clinical features of 149 cases," Multiple Sclerosis Journal, vol. 3, no. 1, pp. 43-46, 1997.

[3] L. B. Krupp, M. Tardieu, M. P. Amato et al., "International Pediatric Multiple Sclerosis Study Group criteria for pediatric multiple sclerosis and immune-mediated central nervous system demyelinating disorders: revisions to the 2007 definitions," Multiple Sclerosis Journal, vol. 19, no. 10, pp. 12611267, 2013.

[4] S. M. Rao, "Neuropsychology of multiple sclerosis," Current Opinion in Neurology, vol. 8, no. 3, pp. 216-220, 1995. 
[5] J. A. Bobholz and S. M. Rao, "Cognitive dysfunction in multiple sclerosis: a review of recent developments," Current Opinion in Neurology, vol. 16, no. 3, pp. 283-288, 2003.

[6] B. L. Banwell and P. E. Anderson, "The cognitive burden of multiple sclerosis in children," Neurology, vol. 64, no. 5, pp. 891-894, 2005.

[7] W. S. MacAllister, A. L. Belman, M. Milazzo et al., "Cognitive functioning in children and adolescents with multiple sclerosis," Neurology, vol. 64, no. 8, pp. 1422-1425, 2005.

[8] M. P. Amato, B. Goretti, A. Ghezzi et al., "Cognitive and psychosocial features of childhood and juvenile MS," Neurology, vol. 70, no. 20, pp. 1891-1897, 2008.

[9] C. Till, R. Ghassemi, B. Aubert-Broche et al., "MRI correlates of cognitive impairment in childhood-onset multiple sclerosis," Neuropsychology, vol. 25, no. 3, pp. 319-332, 2011.

[10] L. Julian, D. Serafin, L. Charvet et al., "Cognitive impairment occurs in children and adolescents with multiple sclerosis: results from a United States network," Journal of Child Neurology, vol. 28, no. 1, pp. 102-107, 2013.

[11] Y. Stern, "Cognitive reserve," Neuropsychologia, vol. 47, no. 10, pp. 2015-2028, 2009.

[12] J. F. Sumowski and V. M. Leavitt, "Cognitive reserve in multiple sclerosis," Multiple Sclerosis Journal, vol. 19, no. 9, pp. 1122-1127, 2013.

[13] L. Pastò, E. Portaccio, B. Goretti et al., "The cognitive reserve theory in the setting of pediatric-onset multiple sclerosis," Multiple Sclerosis Journal, vol. 22, no. 13, pp. 1741-1749, 2016.

[14] S. R. Kesler, H. F. Adams, C. M. Blasey, and E. D. Bigler, "Premorbid intellectual functioning, education, and brain size in traumatic brain injury: an investigation of the cognitive reserve hypothesis," Applied Neuropsychology, vol. 10, no. 3, pp. 153-162, 2003.

[15] J. E. Farmer, S. M. Kanne, J. S. Haut, J. Williams, B. Johnstone, and K. Kirk, "Memory functioning following traumatic brain injury in children with premorbid learning problems," Developmental Neuropsychology, vol. 22, no. 2, pp. 455-469, 2002.

[16] M. P. Amato, B. Goretti, A. Ghezzi et al., "Cognitive and psychosocial features in childhood and juvenile MS two-year follow-up," Neurology, vol. 75, no. 13, pp. 1134-1140, 2010.

[17] M. P. Amato, B. Goretti, A. Ghezzi et al., "Neuropsychological features in childhood and juvenile multiple sclerosis five-year follow-up," Neurology, vol. 83, no. 16, pp. 1432-1438, 2014.

[18] W. S. MacAllister, C. Christodoulou, M. Milazzo, and L. B. Krupp, "Longitudinal neuropsychological assessment in pediatric multiple sclerosis," Developmental Neuropsychology, vol. 32, no. 2, pp. 625-644, 2007.

[19] L. E. Charvet, E. H. O'Donnell, A. L. Belman et al., "Longitudinal evaluation of cognitive functioning in pediatric multiple sclerosis: report from the US pediatric multiple sclerosis network," Multiple Sclerosis Journal, vol. 20, no. 11, pp. 1502-1510, 2014.

[20] B. Hosseini, D. B. Flora, B. L. Banwell, and C. Till, "Age of onset as a moderator of cognitive decline in pediatric-onset multiple sclerosis," Journal of the International Neuropsychological Society, vol. 20, no. 08, pp. 796-804, 2014.

[21] C. Till, N. Racine, D. Araujo et al., "Changes in cognitive performance over a 1-year period in children and adolescents with multiple sclerosis," Neuropsychology, vol. 27, no. 2, pp. 210-219, 2013.

[22] N. F. Baruch, E. H. O'Donnell, B. I. Glanz et al., "Cognitive and patient-reported outcomes in adults with pediatric-onset multiple sclerosis," Multiple Sclerosis Journal, vol. 22, no. 3, pp. 354-361, 2016.

[23] M. F. Green, D. L. Penn, R. Bentall et al., "Social cognition in schizophrenia: an NIMH workshop on definitions, assessment, and research opportunities," Schizophrenia Bulletin, vol. 34, no. 6, pp. 1211-1220, 2008.

[24] R. Adolphs, "Cognitive neuroscience: cognitive neuroscience of human social behavior," Nature Reviews Neuroscience, vol. 4, no. 3, pp. 165-178, 2003.

[25] L. E. Charvet, R. E. Cleary, K. Vazquez, A. Belman, and L. B. Krupp, "Social cognition in pediatric-onset multiple sclerosis (MS)," Multiple Sclerosis Journal, vol. 20, no. 11, pp. 14781484, 2014.

[26] M. Jehna, C. Langkammer, M. Wallner-Blazek et al., "Cognitively preserved MS patients demonstrate functional differences in processing neutral and emotional faces," Brain Imaging and Behavior, vol. 5, no. 4, pp. 241-251, 2011.

[27] J. Cotter, J. Firth, C. Enzinger et al., "Social cognition in multiple sclerosis, a review and meta-analysis," Neurology, vol. 87, no. 16, pp. 1727-1736, 2016.

[28] E. Portaccio, B. Goretti, S. Lori et al., "The brief neuropsychological battery for children: a screening tool for cognitive impairment in childhood and juvenile multiple sclerosis," Multiple Sclerosis Journal, vol. 15, no. 5, pp. 620-626, 2009.

[29] A. M. Smerbeck, J. Parrish, D. Serafin et al., "Visual-cognitive processing deficits in pediatric multiple sclerosis," Multiple Sclerosis Journal, vol. 17, no. 4, pp. 449-456, 2011.

[30] L. E. Charvet, R. Beekman, N. Amadiume, A. L. Belman, and L. B. Krupp, "The symbol digit modalities test is an effective cognitive screen in pediatric onset multiple sclerosis (MS)," Journal of the Neurological Sciences, vol. 341, no. 1-2, pp. 7984, 2014.

[31] C. Till, C. Ho, A. Dudani, D. García-Lorenzo, D. L. Collins, and B. L. Banwell, "Magnetic resonance imaging predictors of executive functioning in patients with pediatric-onset multiple sclerosis," Archives of Clinical Neuropsychology, vol. 27, no. 5, pp. 495-509, 2012.

[32] A. Bethune, V. Tipu, J. G. Sled et al., "Diffusion tensor imaging and cognitive speed in children with multiple sclerosis," Journal of the Neurological Sciences, vol. 309, no. 1-2, pp. 68-74, 2011.

[33] C. Till, A. Deotto, V. Tipu et al., "White matter integrity and math performance in pediatric multiple sclerosis: a diffusion tensor imaging study," NeuroReport, vol. 22, no. 18, pp. 1005-1009, 2011.

[34] D. Timmann, J. Drepper, M. Frings et al., "The human cerebellum contributes to motor, emotional and cognitive associative learning. A review," Cortex, vol. 46, no. 7, pp. 845-857, 2010.

[35] K. Weier, C. Till, V. Fonov et al., "Contribution of the cerebellum to cognitive performance in children and adolescents with multiple sclerosis," Multiple Sclerosis Journal, vol. 22, no. 5, pp. 599-607, 2016.

[36] M. A. Rocca, M. Absinta, M. P. Amato et al., "Posterior brain damage and cognitive impairment in pediatric multiple sclerosis," Neurology, vol. 82, no. 15, pp. 1314-1321, 2014.

[37] F. Paul, "Pathology and MRI: exploring cognitive impairment in MS," Acta Neurologica Scandinavica, vol. 134, Supplement 200, pp. 24-33, 2016.

[38] G. C. DeLuca, R. L. Yates, H. Beale, and S. A. Morrow, "Cognitive impairment in multiple sclerosis: clinical, radiologic and pathologic insights," Brain Pathology, vol. 25, pp. 79-98, 2015. 


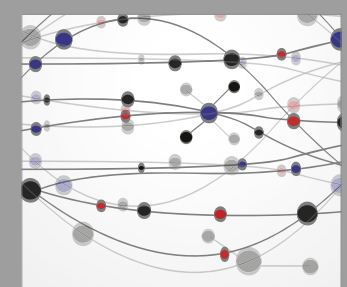

The Scientific World Journal
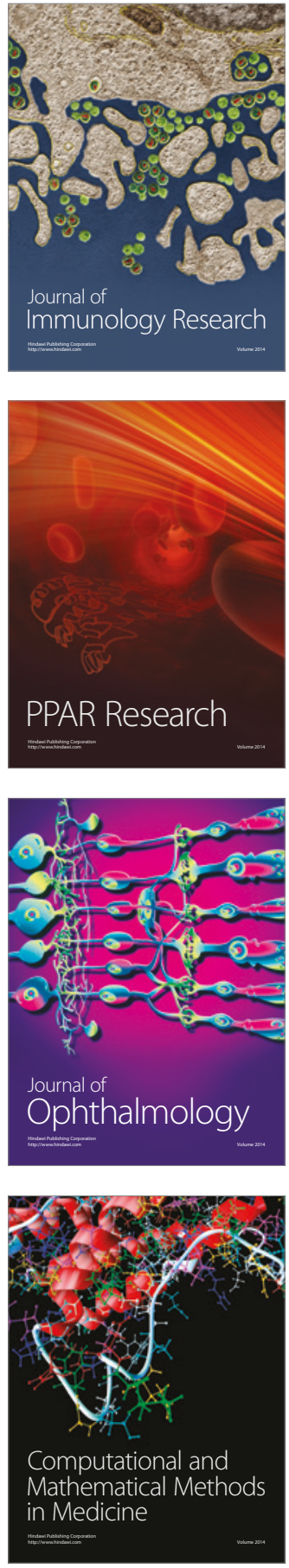

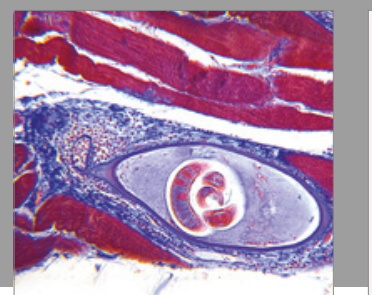

Gastroenterology Research and Practice
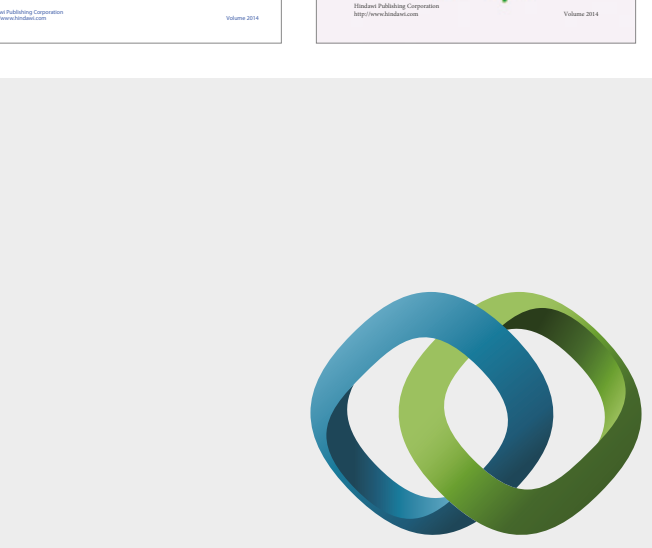

\section{Hindawi}

Submit your manuscripts at

https://www.hindawi.com
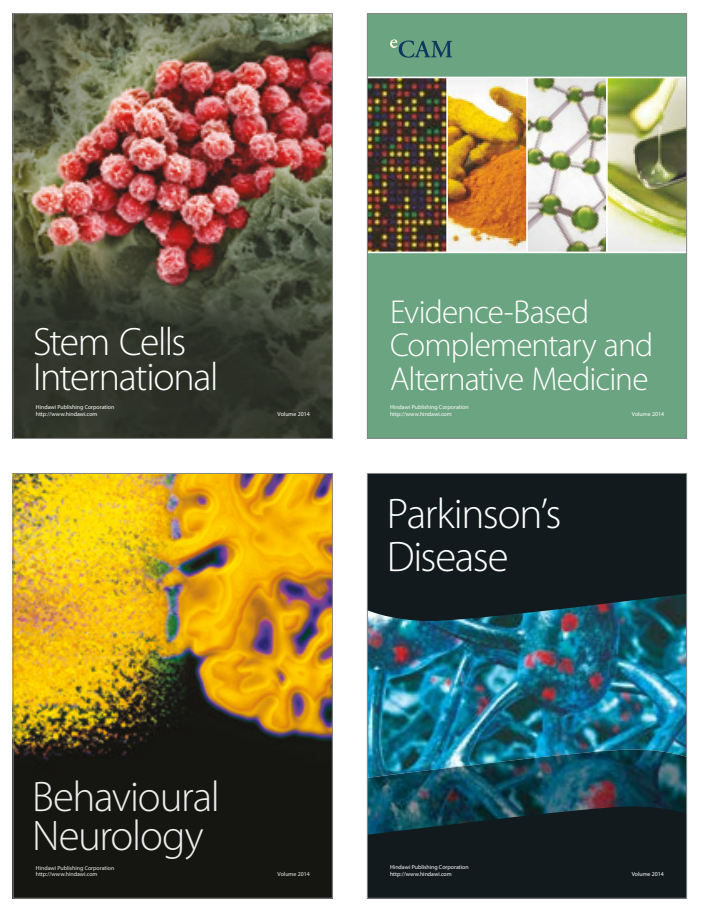
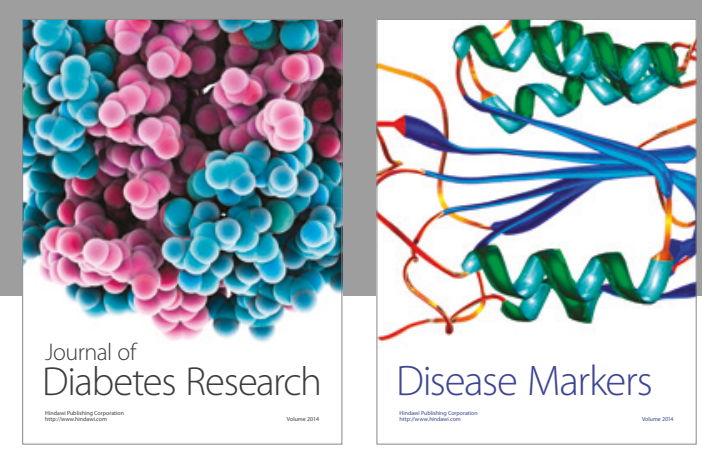

Disease Markers
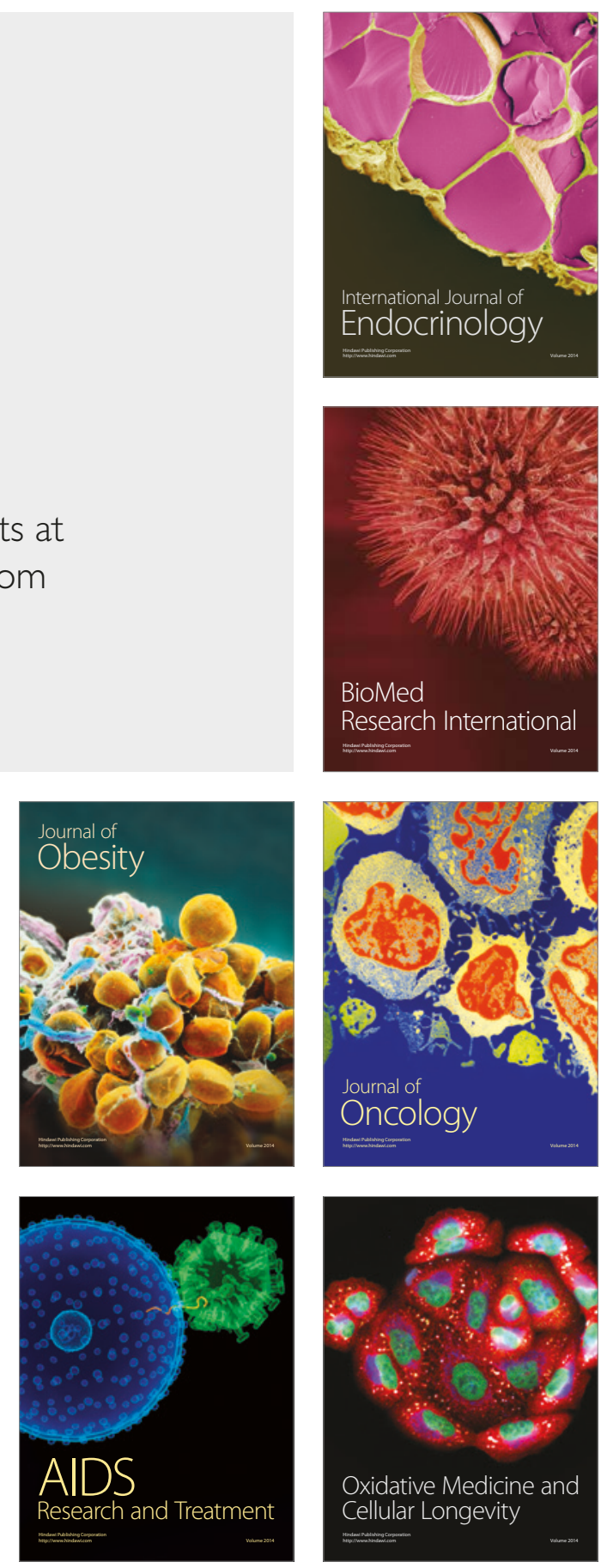